EPJ Web of Conferences 52, 09003 (2013)

DOI: 10.1051/epjconf/20135209003

(C) Owned by the authors, published by EDP Sciences, 2013

\title{
Influence of hadronic interaction models and the cosmic ray spectrum on the high-energy atmospheric muon and neutrino flux
}

\author{
Anatoli Fedynitch $^{1, a}$, Julia Becker Tjus ${ }^{2}$, Paolo Desiati ${ }^{3}$ \\ ${ }^{1}$ CERN/KIT, Switzerland/Germany \\ ${ }^{2}$ Ruhr-Universität Bochum, Germany \\ ${ }^{3}$ WIPAC, Univ. Wisconsin, Madison, USA
}

\begin{abstract}
The recent observations of muon charge ratio up to about $10 \mathrm{TeV}$ and of atmospheric neutrinos up to energies of about $400 \mathrm{TeV}$ has triggered a renewed interest into the high-energy interaction models and cosmic ray primary composition. A reviewed calculation of lepton spectra produced in cosmic ray induced extensive air showers is carried out with a primary cosmic ray spectrum that fits the latest direct measurements below the knee. In order to achieve this, we used a full Monte Carlo method to derive the inclusive differential spectra (yields) of muons, muon neutrinos and electron neutrinos at the surface for energies between $80 \mathrm{GeV}$ and hundreds of PeV. Using these results the differential flux and the flavor ratios of leptons were calculated. The air shower simulator CORSIKA 6.990 was used for showering and propagation of the secondary particles through the atmosphere, employing the established high energy hadronic interaction models SIBYLL 2.1, QGSJet-01 and QGSJet-II-03. We show that the performance of the interaction models allows makes it possible to predict the spectra within experimental uncertainties, while SIBYLL generally yields a higher flux at the surface than the QGSJet models. The calculation of the flavor and charge ratios has lead to inconsistent results, mainly in fluenced by the different representations of the $\mathrm{K} / \pi$ ratio within the models. The influence of the knee of cosmic rays is reflected in the secondary spectra at energies between 100 and $200 \mathrm{TeV}$. Furthermore, we could quantify systematic uncertainties of atmospheric muon- and neutrino fluxes, associated to the models of the primary cosmic ray spectrum and the interaction models. For most recent parametrizations of the cosmic ray primary spectrum, atmospheric muons can be determined with an uncertainty smaller than $+15 /-13 \%$ of the average flux. Uncertainties of the muon and electron neutrino fluxes can be calculated within an average error of $+32 /-22 \%$ and $+25 /-19 \%$, respectively. See the published paper [1].
\end{abstract}

\section{References}

[1] Fedynitch, A. et al, arXiv:1206.6710 [astro-ph.HE], June 2012.

a anatoli.fedynitch@cern.ch

This is an Open Access article distributed under the terms of the Creative Commons Attribution License 2.0, which permits unrestricted use, distribution, and reproduction in any medium, provided the original work is properly cited. 
EPJ Web of Conferences

Slides

The slides of the talk can be found on the website of the symposium ISVHECRI 2012: https://indico.desy.de/conferenceOtherViews.py?view=standard\& confId $=4594$ 